\title{
Interleaving Energy-Conservation Mode (IECM) Control in Single-Inductor Dual-Output (SIDO) Step-Down Converters With 91\% Peak Efficiency
}

\author{
Yu-Huei Lee, Student Member, IEEE, Yao-Yi Yang, Student Member, IEEE, \\ Shih-Jung Wang, Student Member, IEEE, Ke-Horng Chen, Senior Member, IEEE, Ying-Hsi Lin, \\ Yi-Kuang Chen, and Chen-Chih Huang
}

\begin{abstract}
The proposed single-inductor dual-output (SIDO) converter with interleaving energy-conservation mode (IECM) control is designed using $65 \mathrm{~nm}$ technology to power the ultra-wide band (UWB) system. The energy-conservation mode (ECM) control generates four different energy delivery paths for dual buck outputs with only one inductor. In addition, the superposition technique is used to achieve a minimized inductor current level. The average inductor current is equal to the summation of two output loads. Moreover, the IECM control activates the interleaving operation through the current interleaving mechanism to provide large driving capability as well as to reduce the output voltage ripple. As a result, $91 \%$ peak efficiency is derived and the output voltage ripple appears notably minimized by $50 \%$ using current interleaving at heavy load. The test chip occupies $1.44 \mathrm{~mm}^{2}$ in $65 \mathrm{~nm}$ CMOS and integrates with a three-dimensional (3-D) architecture for inductor integration.
\end{abstract}

Index Terms-Current interleaving, DC-DC converter, energy delivery path, output voltage ripple, power conversion efficiency, single-inductor dual-output (SIDO) converter, ultra-wide band (UWB) system.

\section{INTRODUCTION}

$\mathbf{I}$ $\mathrm{N}$ battery-powered mobile systems, a power IC should simultaneously have high efficiency, satisfactory regulation, small volume, and robustness. These characteristics are essential in ultra-wide band (UWB) applications, such as the wireless universal serial bus (USB) that has the advantage of speed over other wireless technologies. The single-inductor dual-output (SIDO) converter [1]-[10] has the capability to provide two high-quality output voltages for different function blocks by using a single inductor. It can reduce the print-circuit-board (PCB) area compared to the single-inductor single-output (SISO) converter [11]-[15] when multi-output supply voltage

Manuscript received August 20, 2010; revised November 15, 2010; accepted December 18, 2010. Date of publication March 03, 2011; date of current version March 25, 2011. This paper was approved by Guest Editor Makoto Nagata. This work was supported by the National Science Council, Taiwan, under Grant NSC 97-2221-E-009-172 and Grant NSC 97-2220-E-009-027.

Y.-H. Lee, Y.-Y. Yang, S.-J. Wang, and K.-H. Chen are with the Institute of Electrical Control Engineering, National Chiao Tung University, Hsinchu 30010, Taiwan (e-mail: khchen@cn.nctu.edu.tw).

Y.-H. Lin, Y.-K. Chen, and C.-C. Huang are with the Realtek Semiconductor Corporation, Hsinchu, Taiwan.

Color versions of one or more of the figures in this paper are available online at http://ieeexplore.ieee.org.

Digital Object Identifier 10.1109/JSSC.2011.2108850 is requested in power management. The comparator-controlled technique with a simple control scheme for power distribution can minimize power consumption to improve efficiency [1], [2], but it sacrifices regulation performance and needs to be redeemed by a post-regulator. The discontinuous conduction mode (DCM) [3] control can distribute the energy into the two outputs of SIDO converter to minimize the cross regulation. However, at heavy loads, an increase in inductor peak current causes a large output voltage ripple. The tri-state control [15] utilizes the freewheel stage to reset the inductor current to a default value at the beginning of every switching cycle. The pseudo-continuous conduction mode (PCCM) [4] combines the advantages of continuous conduction mode (CCM) and DCM, but results in large power loss due to high inductor current level. In addition, to improve efficiency, the energy delivery paths must be well arranged [5]-[7]. Thus, the number of power switches can be decreased [8], but the flexibility is reduced because the outputs must include at least one step-up regulation to release the inductor current for system stability. The energy distribution method in [9] leads to cross talk effect since the inductor charging scheme is controlled by the load condition from one specific output. Fortunately, the CCM operation in [10] solves transient cross regulation and voltage spike by using a flying capacitor. However, the bulk capacitor increases the cost in a real system application.

In conclusion, the essential design issues of the SIDO converter are low output voltage ripple, minimized cross regulation, and high power conversion efficiency. To meet the power requirements of UWB system, the proposed SIDO converter adopts the buck operation that steps down the battery power to dual low voltages. The interleaving energy-conservation mode (IECM) control can achieve the power management function through the demand of four power switches. These four power switches in one SIDO converter constitute the proper energy delivery paths for dual step-down outputs. In addition, a minimum inductor current level is maintained in the proposed control to improve efficiency and provide low ripple, minimized cross regulation, and step-down outputs with a compact 3-D package at the same time.

Fig. 1 shows the proposed SIDO modules constituting the power management function to supply the UWB system with different operation modes. The integration of the two SIDO modules can supply four distinct output voltages, namely, $V_{O A}$, 

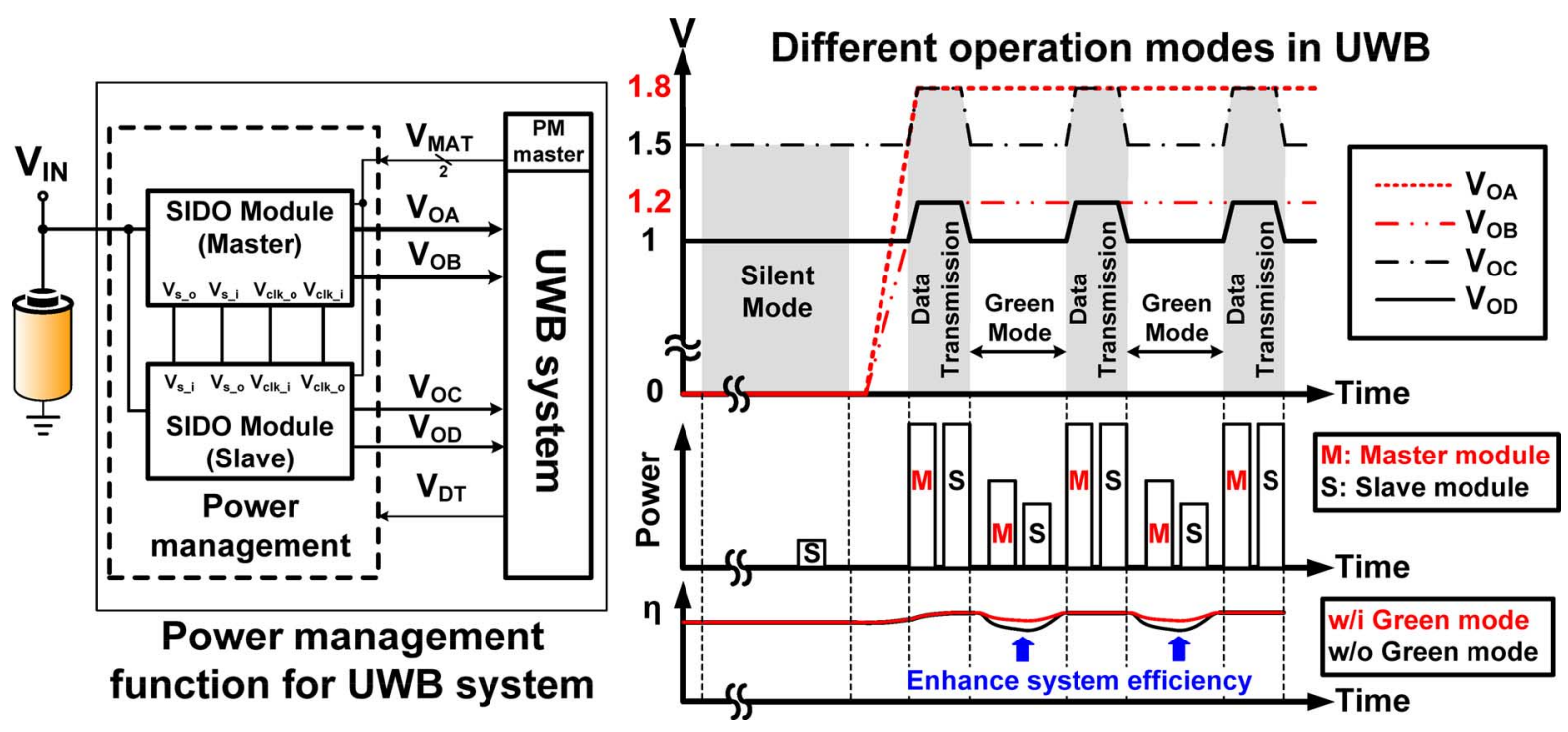

Fig. 1. The proposed SIDO modules form the power management function to supply the UWB system with different operation modes for enhancing the total system efficiency containing power management and UWB.

$V_{O B}, V_{O C}$, and $V_{O D}$. The two-bit signal $V_{M A T}$ is used to indicate the supply function of the two SIDO modules. The singlephase operation of the two SIDO modules is achieved by the energy-conservation mode (ECM) control, whereas the interleaving-ECM (IECM) control is activated in the data transmission period enabled by the signal $V_{D T}$ generated from the UWB system. The IECM control also provides large supply power and minimizes output voltage ripple through the current interleaving mechanism.

Power management can vary the supply voltages to meet the distinct power requests in the UWB system with different operation modes according to the throughput constraint. During the silent mode, there are no data in the vacant transmission time slot. Thus, the power request is low, and only the slave SIDO module operates in order to reduce power dissipation as well as shutdown the master SIDO module to extend battery life. The voltages $V_{O C}$ and $V_{O D}$ generated from the slave module are set to $1.5 \mathrm{~V}$ and $1 \mathrm{~V}$, respectively, to supply the awaiting circuit in the UWB system. The master module is activated in the upcoming period of data transmission. The output voltages, $V_{O A}$ and $V_{O B}$, are raised to $1.8 \mathrm{~V}$ and $1.2 \mathrm{~V}$ to supply the radio frequency (RF) circuits and digital circuits, respectively. The voltages, $V_{O C}$ and $V_{O D}$, yielded from the slave module can be raised up to $1.8 \mathrm{~V}$ and $1.2 \mathrm{~V}$, respectively, to form an interleaving operation for large driving capability. The current balance mechanism can guarantee the current matching to ensure the equivalent power delivery ability of the two SIDO modules. Aside from this, a green-mode operation is implemented to decrease power dissipation of UWB. According to the UWB protocol for supporting multiple low-power modes, the standby and hibernate modes can be used during data transmissions. Thus, the slave SIDO module can scale down the output voltages, $V_{O C}$ and $V_{O D}$, back to $1.5 \mathrm{~V}$ and $1 \mathrm{~V}$, respectively, for some low-power blocks. In the proposed power management design, green-mode would be activated if the interval between two data transmission period in UWB is shorter than $1 \mathrm{~ms}$ since the re-startup procedure of the proposed SIDO module needs the response time about $0.1 \mathrm{~ms}$. Moreover, it can enhance the system efficiency containing the power management and UWB owing to the reduction of the power consumption.

The brief comparison of the power management implementation is listed in Table I. The proposed SIDO module with the single phase ECM control and the interleaving operation derived from IECM is a suitable solution to supply the UWB system under the different operation modes. In this paper, the structure of a SIDO converter is described in Section II. The ECM controlled single-phase and interleaving operations with the IECM control are illustrated in Section III. Detailed circuit implementations of the SIDO converter are discussed in Section IV. Experimental results and the 3-D inductor integration are presented in Section V. Finally, the conclusion is given in Section VI.

\section{Proposed SIDO CONVERTER Structure}

The structure of the proposed SIDO converter is shown in Fig. 2. The control circuits in the proposed SIDO converter are all implemented using $65 \mathrm{~nm}$ deep-submicron devices with a $1.2 \mathrm{~V}$ voltage supply [16], $V_{\text {core }}$. Thus, low power consumption and small silicon area are achieved.

The power stage is composed of four power switches, $M_{S 1}-M_{S 4}$, for the dual step-down outputs. These power switches are implemented by the I/O devices in $65 \mathrm{~nm}$ process for high voltage tolerance. It has four different energy delivery paths to transfer energy from the battery, $V_{\mathrm{IN}}$, to the dual outputs with only one inductor used. Sensor gains are decided by two voltage dividers that contain $R_{A 1}, R_{A 2}, R_{B 1}$, and $R_{B 2}$ for the feedback of output voltage information to the two cascade error amplifiers [16], which yields high DC voltage gain under the low voltage operation. The two error current signals, $I_{E A}$ and $I_{E B}$, generated by voltage-to-current (V-to-I) converters at the output of two EAs, are sent to the ECM controller to decide the duty ratio of the dual outputs. The current $I_{E A B}$, which is the summation of $I_{E A}$ and $I_{E B}$, indicates the peak 
TABLE I

BRIEF COMPARISON OF THE POWER MANAGEMENT IMPLEMENTATIONS FOR UWB SYSTEM

\begin{tabular}{|c|c|c|c|c|}
\hline \multicolumn{2}{|c|}{ Power management for UWB system } & $\begin{array}{c}\text { SISO } \\
\text { (Current-mode) }\end{array}$ & $\begin{array}{c}\text { SIDO } \\
(\text { ECM or IECM) }\end{array}$ & $\begin{array}{c}\text { SIDO } \\
(\mathrm{PCCM})\end{array}$ \\
\hline \multicolumn{2}{|c|}{ Outputs type } & Buck & Buck & Buck \\
\hline \multicolumn{5}{|c|}{ Key Specifications } \\
\hline \multirow{4}{*}{$\begin{array}{c}4 \text { Outputs } \\
\text { (Green-mode) } \\
\text { (Light load) }\end{array}$} & Num. of off-chip inductor & 4 & 2 & 2 \\
\hline & Num. of off-chip capacitor & 4 & 4 & 4 \\
\hline & Output voltage ripple & Small & Small & Medium \\
\hline & Driving capability for outputs & Large & Medium & Medium \\
\hline \multirow{4}{*}{$\begin{array}{c}2 \text { Outputs } \\
\text { (Data transmission) } \\
\text { (Heavy load) }\end{array}$} & Num. of off-chip inductor & 2 & 2 & 1 \\
\hline & Num. of off-chip capacitor & 2 & 2 & 2 \\
\hline & Output voltage ripple & Small & Small & Large \\
\hline & Driving capability for outputs & Large & Large & Medium \\
\hline
\end{tabular}

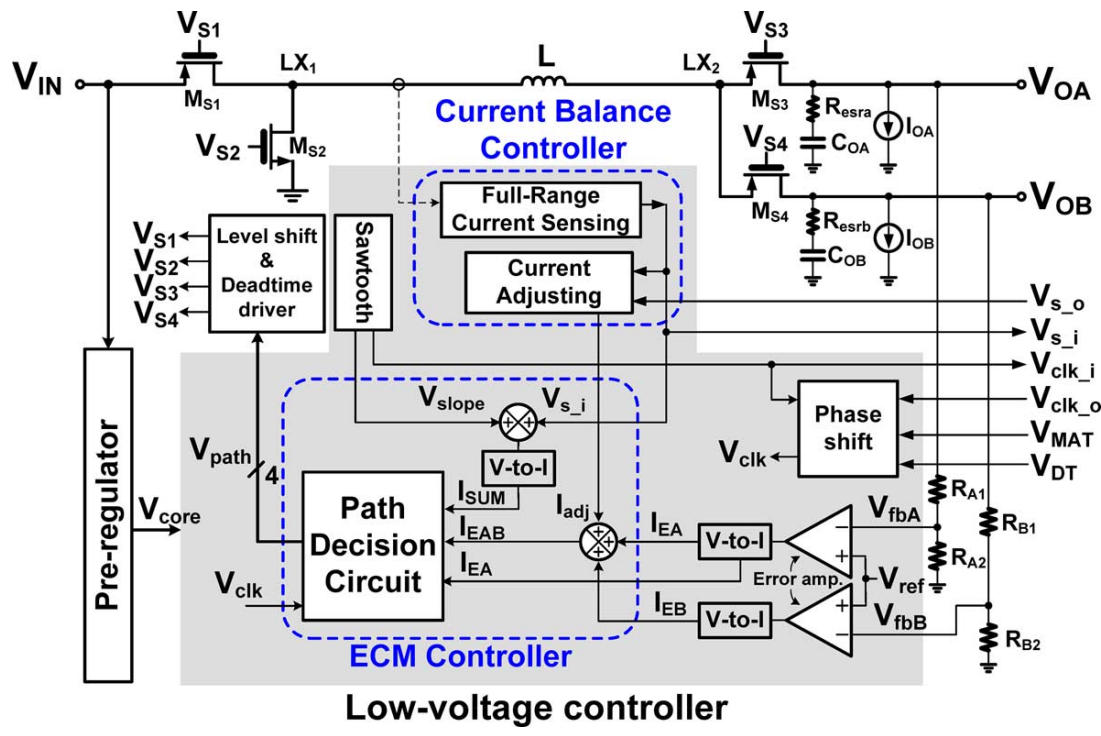

Fig. 2. Structure of the proposed SIDO converter with the IECM control.

inductor current as well as the required energy in one switching cycle through the ECM control. Therefore, by using the ECM controller, the average inductor current is lowered to equal the summation of the two output load currents. In addition, $I_{E A B}$ is adjusted by the current adjusting signal $I_{a d j}$ to ensure the current matching in the IECM control with the interleaving operation. $I_{a d j}$ is generated from the current adjusting circuit in the current balance controller. It can adjust the inductor current levels of each SIDO module in the interleaving operation.

The current sensing signal, $V_{s_{-} i}$, generated from the current sensing circuit is added to a slope compensation signal, $V_{\text {slope }}$, to avoid sub-harmonic oscillation [17]. Hence, the currents, $I_{S U M}, I_{E A B}$, and $I_{E A}$ are used to determine the four-bit signal, $V_{\text {path }}$, in the path decision circuit. Additionally, the current sensing signal $V_{s_{-} o}$, which is derived from the current sensing of the other SIDO module, and $V_{s_{-} i}$ are used to achieve the current balance when the IECM control is activated. Moreover, $V_{c l k_{-} i}$ is generated from the sawtooth circuit, and $V_{c l k_{-} o}$ is derived from the other SIDO converter. Thus, the phase shift circuit can generate system clock $V_{c l k}$ to accomplish the interleaving operation. The level-shift and deadtime driver circuit produces the control signals, $V_{S 1}-V_{S 4}$, from the four-bit signal $V_{\text {path }}$ to drive the power switches due to the use of low-voltage controller. The level-shift circuit can enhance the driving ability of power switches by increasing the gate driving voltages. The deadtime circuit can prohibit the simultaneous on-state to avoid the shoot-through current between power switches $M_{S 1}$ and $M_{S 2}$.

\section{IECM OPERATION}

For high efficiency operation in the UWB system, singlephase and interleaving operations are included in the proposed SIDO converter. Thus, the integration of the two SIDO converters can provide either four different output voltages in the green-mode period or two distinct output voltages with high driving capability in the data transmission period.

\section{A. Single-Phase Operation-ECM Control}

Fig. 3 shows the energy delivery paths of the SIDO converter. The four energy delivery paths, path-I to path-IV, constitute distinct inductor current slopes. Path-I and path-III indicate the inductor charging path and deliver energy from the input to the outputs $V_{O A}$ and $V_{O B}$, respectively. Path-II and path-IV indicate the inductor discharging path and deliver energy from the inductor to the outputs $V_{O A}$ and $V_{O B}$, respectively. The proposed ECM control, which uses one of the combinations of these possible energy delivery paths, can achieve the CCM dual buck operations with a minimum average inductor current level. 


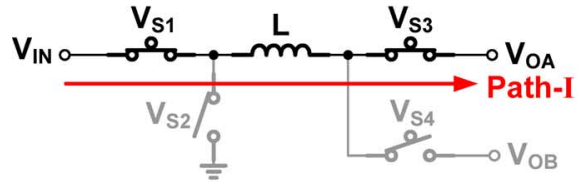

(a)

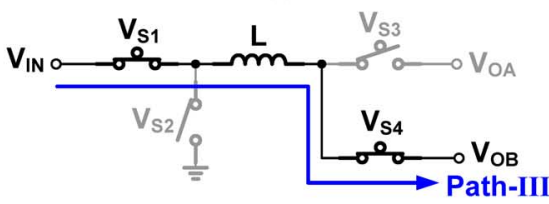

(c)

Fig. 3. Energy delivery paths in the power stage of the SIDO converter.

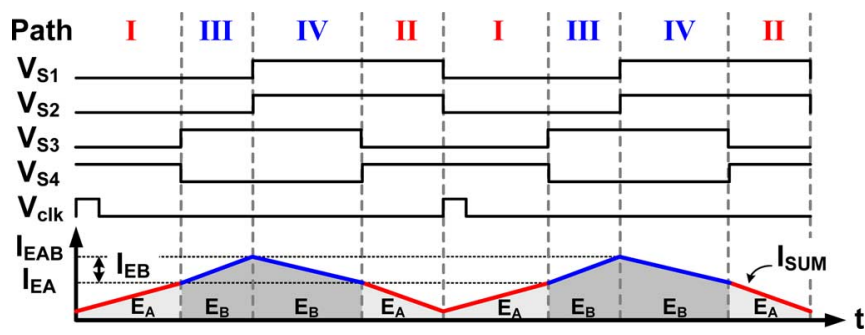

Fig. 4. The time diagram of the proposed ECM control.

Fig. 4 shows the time diagram of the ECM control with the order of path-I, path-III, path-IV, and path-II. This ordered sequence circulates by the triggering of the system clock, $V_{c l k}$. $I_{E A B}$, which is decided by the summation of the two error signals, $I_{E A}$ and $I_{E B}$, indicates the peak inductor current. The error signal $I_{E A}$ determines the transitions from path-I to path-III and from path-IV to path-II. That is, the Path-I and Path-II are decided by $I_{S U M}$ and $I_{E A}$ for the output $V_{O A}$. Similarly, the Path-III and Path-IV are decided by $I_{S U M}$ and $I_{E B}$, which is the difference between $I_{E A B}$ and $I_{E A}$. The ECM control can achieve the separated dual step-down operations with the superposition scheme. Thus, each output can receive battery power in one PWM switching period through the ECM control. Besides, each power switch, $M_{S 1}$ to $M_{S 4}$, would switch twice in the period of one switching cycle time with the ordered energy delivery paths.

Fig. 5 illustrates the inductor current waveform under different load conditions with the ECM control. The combination of the two separated step-down operations in ECM control results in the average inductor current, $I_{L, a v g_{-} E C M}$, to be equal to the summation of the two output loads. The value of $I_{L, a v g_{-} E C M}$ is lower than that of $I_{L, a v g_{-} P C C M}$ derived from the PCCM control, which needs a freewheel stage to regulate the inductor current. As such, the ECM control can yield a low average inductor current level to reduce conduction loss and enhance efficiency by removing the freewheel stage. Moreover, the duty cycle of each regulated output voltage varies depending on the loading in the ECM control. For example, if the load current $I_{O B}$ of the output voltage $V_{O B}$ increases and the load current $I_{O A}$ of the output voltage $V_{O A}$ is fixed, there would be an increase in total output power. Thus, the average inductor current of the ECM control $I_{L, a v g_{-} E C M}$ arises to reflect the

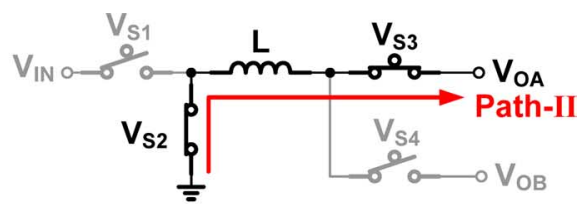

(b)

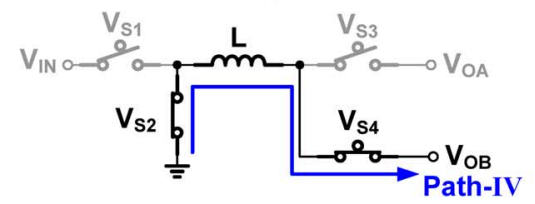

(d)

heavy load condition. The total energy $E_{A}$ for $V_{O A}$ in Fig. 5 must keep constant at both light load and heavy load conditions since $I_{O A}$ would not change. On the other hand, the total energy $E_{B}$ for $V_{O B}$ should be increased to provide a sufficient power owing to the increase of $I_{O B}$. As a result, the periods of path-I and path-II are reduced, but the periods of path-III and path-IV are extended because of the increase in $I_{L, a v g_{E} E C M}$. Moreover, the effect from PVT condition to the proposed ECM control can be minimized since the high gain error amplifiers [16] are used to compensate these variations. They modulate the error signals properly to ensure the correct function of ECM control. Consequently, adequate energy is delivered to the outputs to satisfy the output loads. In other words, the low value of $I_{L, a v g_{-} E C M}$ greatly enhances the power conversion efficiency in ECM control owing to the use of superposition technique. Furthermore, the derivation of the minimum average inductor current level helps reduce the output voltage ripple to better the supply quality of the proposed SIDO converter.

\section{B. Interleaving Operation - Interleaving ECM (IECM) Control}

The increase in load current can cause a large output voltage ripple due to the discontinuous inductor current derived at the output nodes of the SIDO converter. Thus, to better enhance the performance at heavy loads, the IECM control is utilized for a power management function to the UWB system. That is, the interleaving operation focuses on providing a large power supply during the data transmission period. The interleaving scheme for the UWB system is depicted in Fig. 1. When the interleaving operation is enabled during the data transmission period, $V_{O C}$ is paralleled to $V_{O A}$ to provide a large supply power to the RF PA and Mixer circuit in the UWB system. Similarly, $V_{O D}$ is paralleled to $V_{O B}$ to simultaneously deliver energy to the digital circuit in the UWB system. The power management (PM) master in the UWB processor activates the interleaving operation of the two SIDO modules and decides the power management functions according to the demand with different UWB operation modes.

The interconnection of the control signals between the two SIDO modules is also shown in Fig. 1. To achieve current matching and output voltage ripple minimization, some control signals between the two SIDO modules should be adequately 


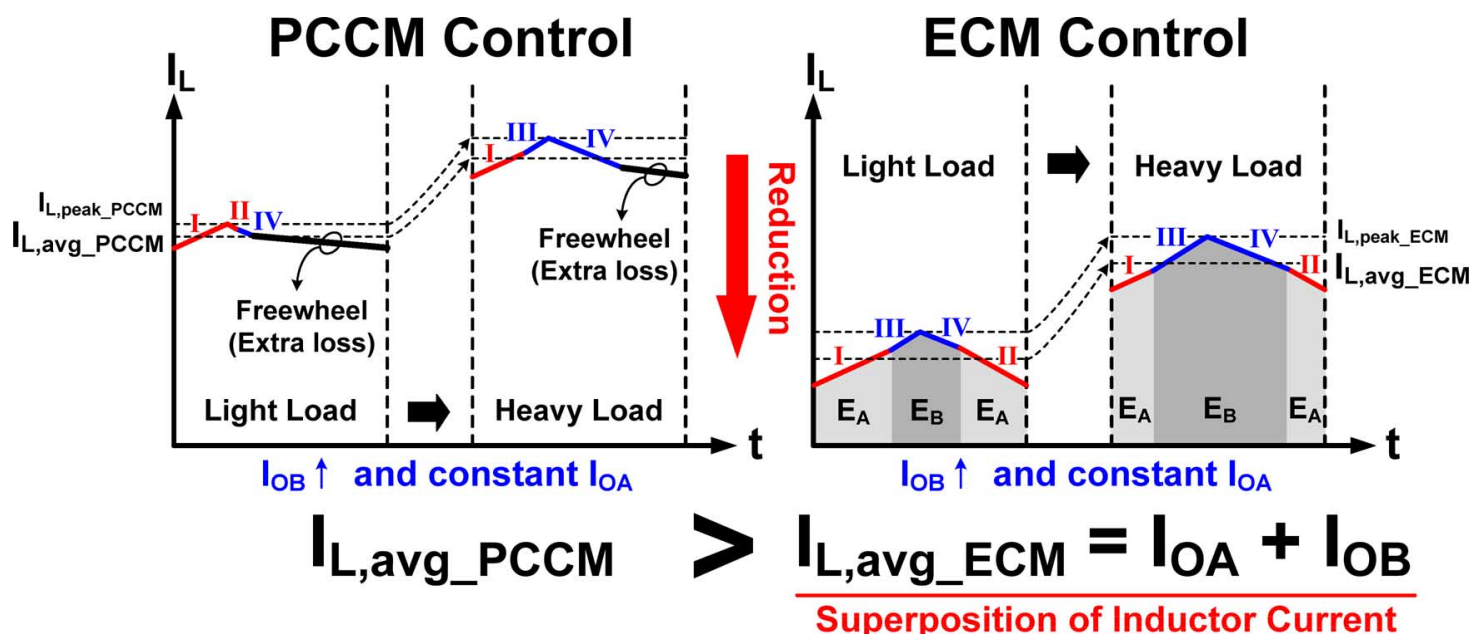

Fig. 5. The inductor current waveform under different load conditions with different control methods.

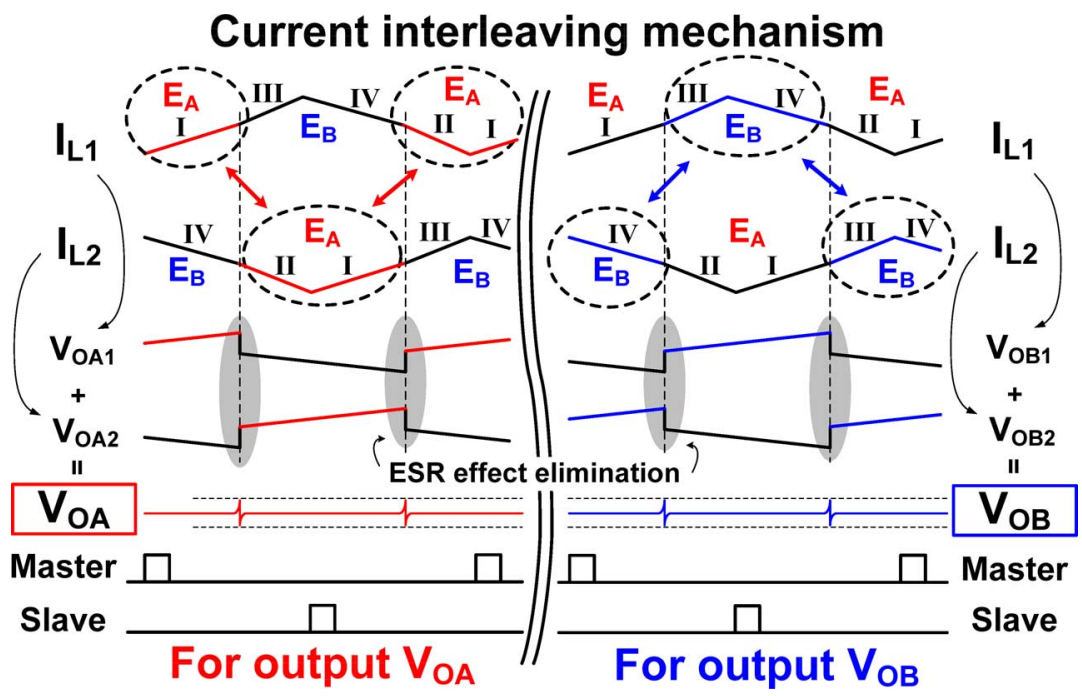

Fig. 6. The current interleaving mechanism in the proposed IECM control during the interleaving operation.

linked together to achieve the interleaving operation. Two signals of $V_{s_{-} i}$ and $V_{s_{-o}}$ generated from different current sensing circuits are used to achieve the current balance. In each SIDO module, the $V_{s_{-} i}$ signal indicates the current sensing signal of the SIDO module itself, whereas the $V_{s_{-O}}$ signal represents the current sensing signal generated from the other SIDO module. Similarly, the signal, $V_{c l k \_i}$ obtained from the SIDO module itself and $V_{c l k_{-} o}$ obtained from the other SIDO module, are utilized to adjust the phase difference between the two SIDO modules.

The detailed current interleaving mechanism in the interleaving operation is shown in Fig. 6. $I_{L 1}$ is the inductor current of the master module, whereas $I_{L 2}$ is the inductor current of the salve module. The single-phase SIDO converter has to provide energy for two output nodes, which results in a discontinuous inductor current at each output nodes. Through the interleaving operation of the two proposed SIDO modules, the two separated inductor currents interleave with each other so that the output current appears to be a continuous function. That is, with the current interleaving mechanism, $V_{O A}$ derives energy from $I_{L 1}$ and $I_{L 2}$ through the different phase opera- tions. It helps inductor current be continuous at the node $V_{O A}$. Furthermore, the existence of the parasitic equivalent series resistance (ESR) in the output capacitor also causes a large output voltage ripple due to the discontinuous inductor current in the single-phase operation. Nevertheless, the interleaving operation can produce the continuous inductor current by current interleaving to minimize the output voltage ripple. The glitches carried out by the ESR can also be cancelled naturally. Additionally, if the load $I_{O A}$ increases during the interleaving operation, the error signals $I_{E A}$ and the peak signals $I_{E A B}$ in each SIDO modules are also increased. Thus, both of the average inductor current in each SIDO module is raised to supply the output load simultaneously. Moreover, the current adjusting circuit in the current balance controller shown in Fig. 2 ensures the current matching in the interleaving operation. The current adjusting signal $I_{a d j}$ is used to slightly adjust the inductor current level of the two SIDO modules for guaranteeing the current matching. As a result, the interleaving operation is a way of suppressing the output voltage ripple as well as of providing high power driving capability to supply the UWB system. 


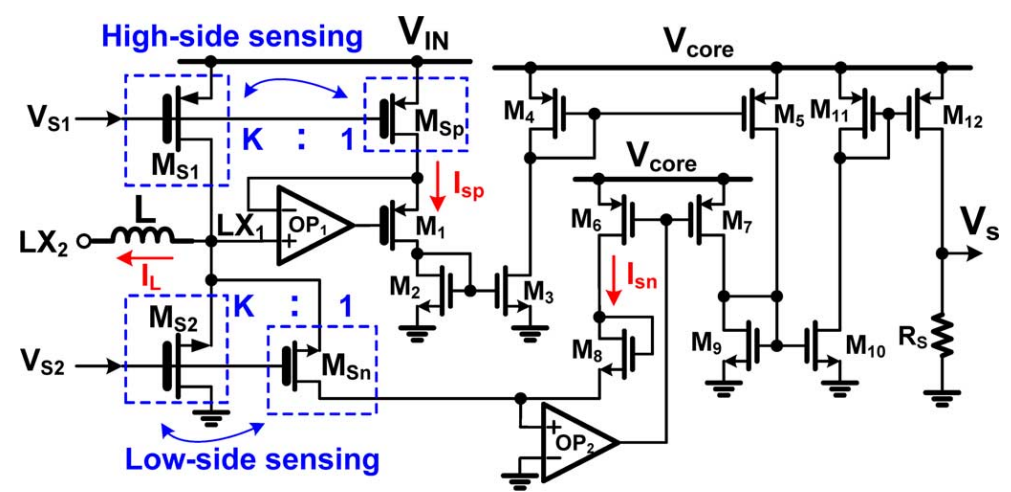

(a)

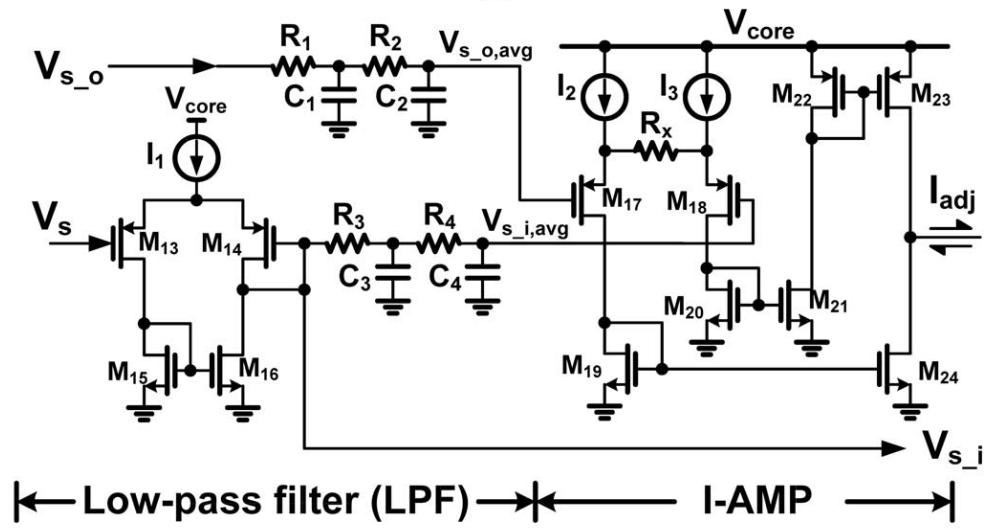

(b)

Fig. 7. The current balance controller circuit. (a) Full-range current sensing circuit. (b) Current adjusting circuit.

\section{CiRCUit IMPLEMENTATION}

\section{A. Current Balance Controller}

Fig. 7 shows the current balance controller containing the full-range current sensing circuit and the current adjusting circuit. The inductor current information is necessary for ECM or IECM control owing to the current-mode operation. The fullrange current sensing is utilized to achieve the four different energy delivery paths with the superposition technique in the proposed SIDO converter.

Fig. 7(a) shows the schematic of the full-range current sensing circuit. $I_{L}$ is the current flowing through the inductor and $\mathrm{K}$ is the sensing factor of both $M_{S 1}$ to $M_{S p}$ and $M_{S 2}$ to $M_{S n}$. These four transistors are all implemented by I/O devices for high voltage tolerance. The transistor $M_{S p}$ produces the sensing current during the turn-on period of the high-side power switch $M_{S 1}$. The source-to-drain voltages of $M_{S 1}$ and $M_{S p}$ are approximately equal because of the closed-loop generated by the operational amplifier $O P_{1}$. Accordingly, the current $I_{s p}$ flowing through the transistors $M_{S p}$ becomes proportional to the current flowing through the $M_{S 1}$, thus achieving the high-side current sensing. Identically, the transistor $M_{S n}$ produces the inductor current information during the low-side turn-on period. The closed-loop generated by the operational amplifier $\mathrm{OP}_{2}$ carries out a sensing current $I_{s n}$ flowing through the transistor $M_{6}$ and the sensing transistor $M_{S n}$. Therefore, the full-range current sensing signal $V_{s}$, which is the voltage across the sensing resistor $R_{s}$, is generated through the summa- tion of the two sensing currents, $I_{s p}$ and $I_{s n}$. The description is shown in (1).

$$
V_{s}=\left(I_{s p}+I_{s n}\right) R_{S}=\frac{I_{L}}{K} R_{S}
$$

Current balance mechanism between the two SIDO modules is implemented to ensure equivalent driven capability when the IECM control is activated for interleaving operation. As shown in Fig. 7(b), the current adjusting circuit, which is composed of the low-pass filter (LPF) and the current amplifier (I-AMP), is adopted to adjust the inductor current level of the two interleaved SIDO modules. The full-range current sensing signal $V_{s}$ is buffered for transmission to the other SIDO module through the signal, $V_{s_{-} i}$. Similarly, the sensing signal $V_{s_{-} o}$ is generated from the other SIDO module. The buffer stage eliminates the load effect and also filters out the switching noise. Hence, these two full-range current sensing signals are filtered by the LPF to obtain the average inductor current of each SIDO module in the interleaving operation. Additionally, the capacitors, $C_{1}$ to $C_{4}$, in the LPF are implemented by the capacitor multiplier technique to achieve the fully on-chip integration [11]. The average inductor current information of the two SIDO modules, $V_{s_{-} i, a v g}$ and $V_{s_{-}, a v g}$, are sent to the I-AMP circuit to generate an adjusting current $I_{a d j}$ to achieve the current balance when the IECM control is activated. An auxiliary resistor $R_{x}$ in the I-AMP circuit can strengthen the linearity of $I_{a d j}$. The illustration of the absolute value of $I_{a d j}$ is shown in (2).

$$
\left|I_{a d j}\right|=2 \cdot \frac{V_{s_{-}, a v g}-V_{s_{-}, a v g}}{R_{x}} .
$$




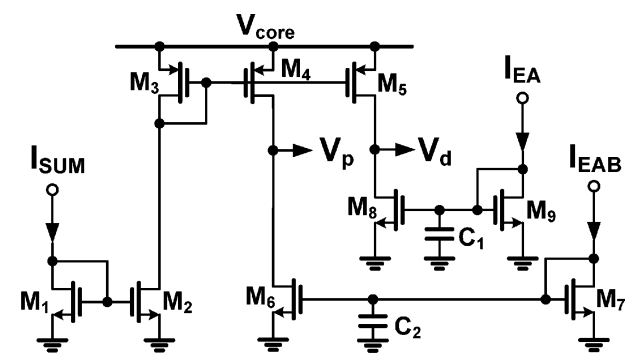

(a)

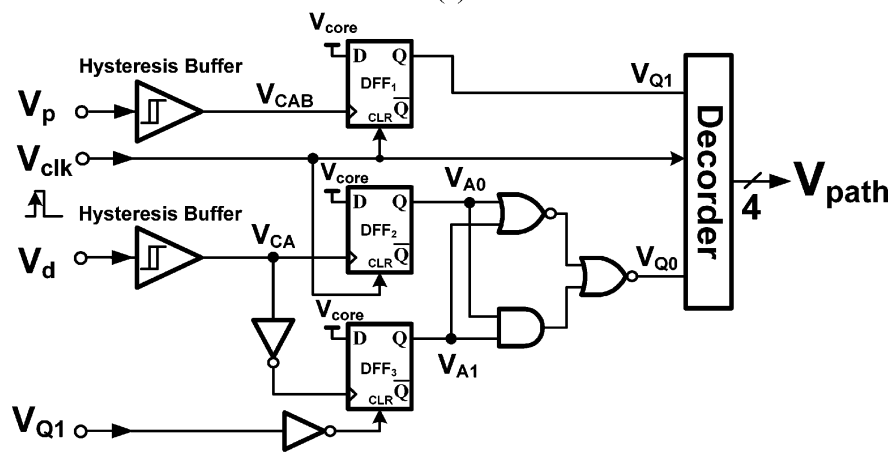

(b)

Fig. 8. The path decision circuit. (a) Current comparator. (b) Path decision logic.

\section{B. ECM Controller}

As shown in Fig. 8, the path decision circuit in the ECM controller, which contains the current comparator and the path decision logic, generates duty cycles to the two outputs in one SIDO module. The current comparator shown in Fig. 8(a) operates with $I_{E A}, I_{E A B}$ and $I_{S U M}$. The values of $V_{p}$ and $V_{d}$ used to indicate the transition of energy delivery paths are decided by the intersection of $I_{S U M}$ with $I_{E A B}$ and $I_{E A}$, respectively.

The path decision logic depicted in Fig. 8(b) generates the four-bit $V_{\text {path }}$ signal to achieve the energy delivery paths. The hysteresis buffer enhances the noise immunity of the current comparator to get a robust ECM control. As the illustration of timing diagram shows in Fig. 9, $V_{c l k}$ triggers the PWM switching as well as energy delivery path-I. $V_{C A}$ is set to high when $I_{S U M}$ intersects $I_{E A}$ during the inductor charging period. Thus, the energy delivery path is changed from path-I to path-III. In addition, $V_{C A B}$ is triggered to realize the path transition from path-III to path-IV when $I_{S U M}$ reaches $I_{E A B}$. Moreover, path-II is carried out when $I_{S U M}$ intersects $I_{E A}$ again. The energy delivery path is swapped from path-IV to path-II with a negative edge voltage trigger provided by $V_{C A}$. Finally, path-II appears until the occurrence of the next $V_{c l k}$, thus initialing the next PWM switching. The stages of the current comparator are minimized by using D-flip-flops due to the path decision logic. Furthermore, $V_{Q 1}$ and $V_{Q 0}$ indicate the energy delivery paths with binary code, which can be decoded through the decoder to generate a four-bit $V_{\text {path }}$ signal to achieve the ECM control.

\section{Phase Shift Circuit}

The IECM control interconnects the two SIDO modules to provide a large power supply and minimize the output voltage ripple simultaneously. Thus, the system clocks in the two SIDO

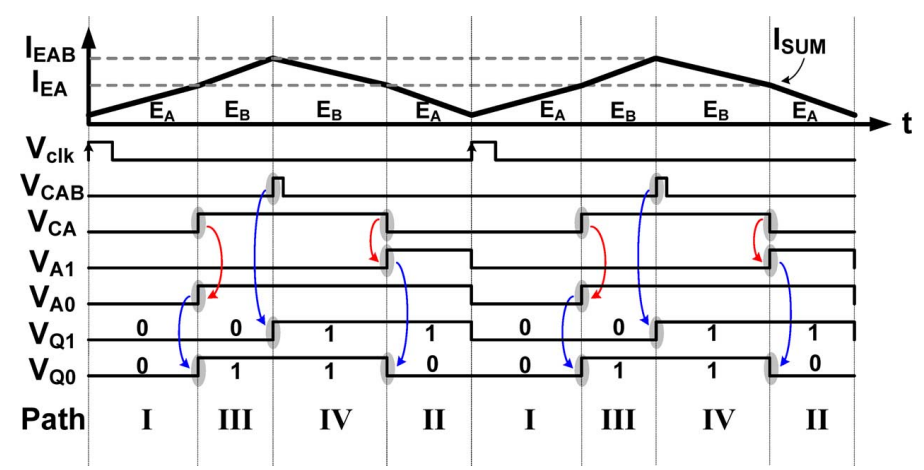

Fig. 9. Timing diagram of the path decision circuit.

modules must be out of phase to achieve the current interleaving mechanism effectively. The proposed phase shift circuit shown in Fig. 10 produces the system clock $V_{c l k}$, which is relative to the interconnected signals, $V_{c l k_{-} i}$ and $V_{c l k_{-o}}$, generated from the two distinct SIDO modules. In addition, once $V_{D T}$ activates the interleaving operation, $V_{M A T}$ from the PM master in the UWB system will indicate either the master or slave function to each SIDO modules.

Fig. 11 shows the timing diagram of the phase shift circuit. In a single-phase operation, $V_{D T}$ is set to low, and $V_{c l k}$ is derived from $V_{c l k \_i}$ through the selection of a multiplexer. $V_{c l k}$ is also directly obtained from $V_{c l k \_i}$ when one SIDO converter is utilized as the power management master in the interleaving operation. The $V_{M A T}$ signal is set to high to indicate the master power management module. However, the SIDO module is pronounced a slave module if the $V_{M A T}$ is set to low. $V_{c l k}$ is generated from the two shift-cells in order to obtain an out-of-phase operation to reduce the output voltage ripple. That is, $V_{c l k_{-} o}$ is linked to the system clock $V_{c l k}$ of the master module through the interconnections and generates the signals $V_{K}$ and $V_{K B}$ by the frequency divider. $V_{K}$ and $V_{K B}$ trigger the shift-cells and acquire the sawtooth waveforms, $V_{c_{-} 1}$ and $V_{c_{-} 2}$. When the signal $V_{K}$ is set to high, the switch $M_{1}$ in the shift-cell $l_{1}$ circuit is on in order to charge the capacitor $C_{1}$ with a fixed current $I_{B}$. On the contrary, $C_{1}$ is discharged with the current $2 I_{B}$, until the $V_{c_{-} 1}$ reverts to the value of $V_{L}$. The amplitude of the sawtooth waveform is $V_{p p}$. This operation is illustrated in (3).

$$
T=\frac{C_{i} V_{p p}}{I_{B}} \text { and } t_{i}=\frac{C_{i} V_{p p}}{2 I_{B}} \text { where } i=1,2 .
$$

The D-flip-flop detects when the discharge period is over. $V_{T 1}$ is set to high at the half period location with $V_{c l k_{-} o}$. Similarly, $V_{T 2}$ is produced through the identical operation mechanism in the shift-cell $l_{2}$ circuit triggered by $V_{K B}$. By the equations shown in (3), $t_{1}$ and $t_{2}$ are equal to $T / 2$, which is used to assure the interleaving operation. Finally, $V_{c l k}$ is carried out by the OR operation from $V_{T 1}$ and $V_{T 2}$ in the slave SIDO module to achieve the interleaving operation. Moreover, the bottom plates of capacitor $C_{1}$ in each shift-cell are fixed to a constant voltage $V_{A}$ decided by the buffer stage, which is composed of the operational amplifier and the transistor $M_{n}$. This design helps avoid the voltage nonlinear phenomenon in the charging and discharging periods owing to the MOSFET as a capacitor. The interleaving operation with IECM control is shown in Fig. 12 that demonstrate the 


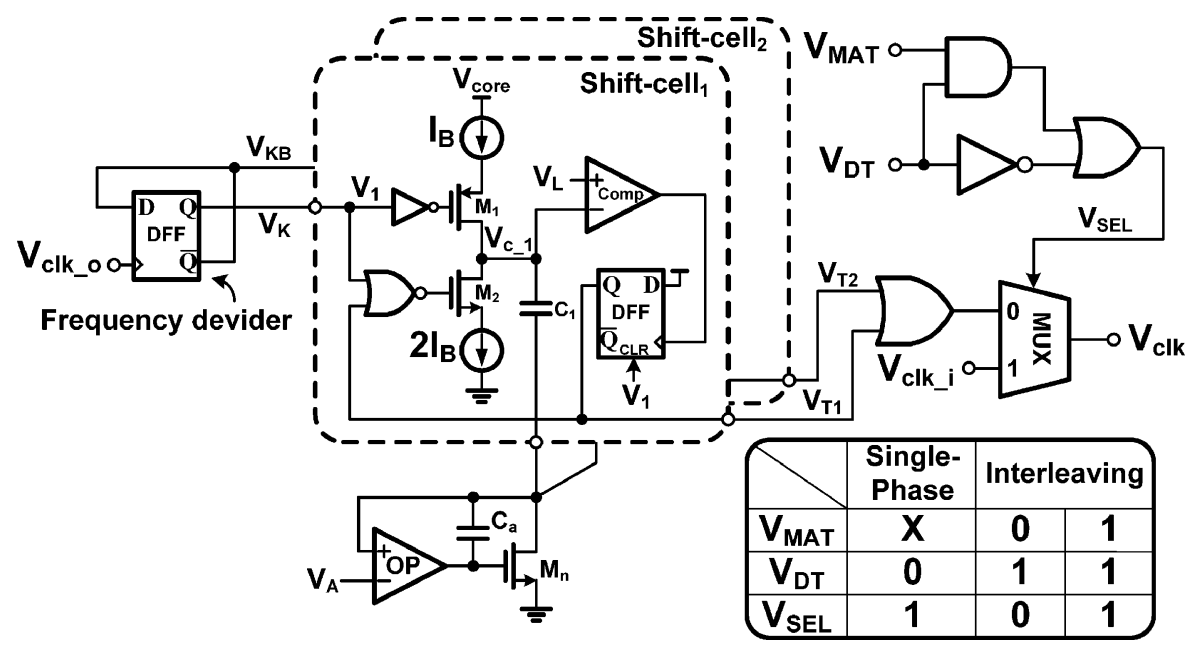

Fig. 10. The phase shift circuit for interleaving operation.

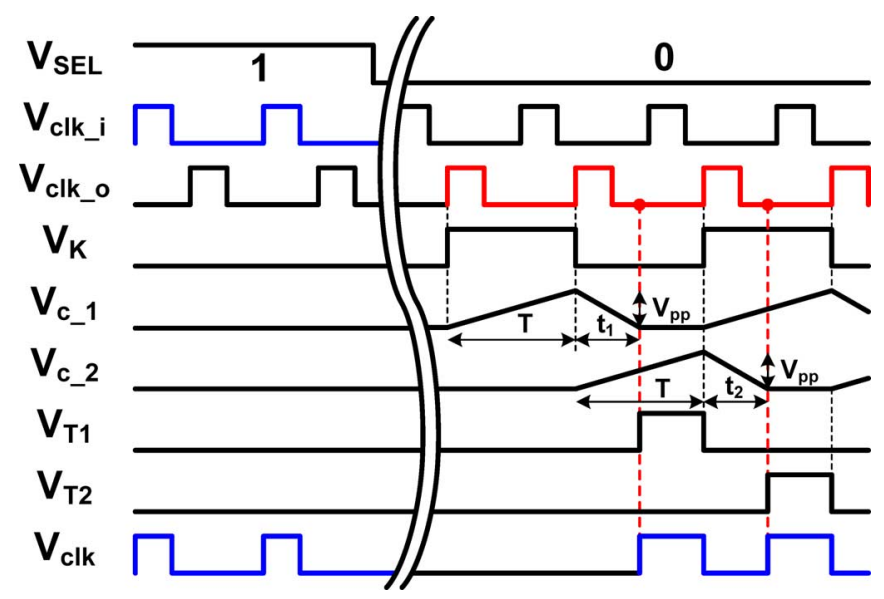

Fig. 11. Timing diagram of the phase shift circuit.

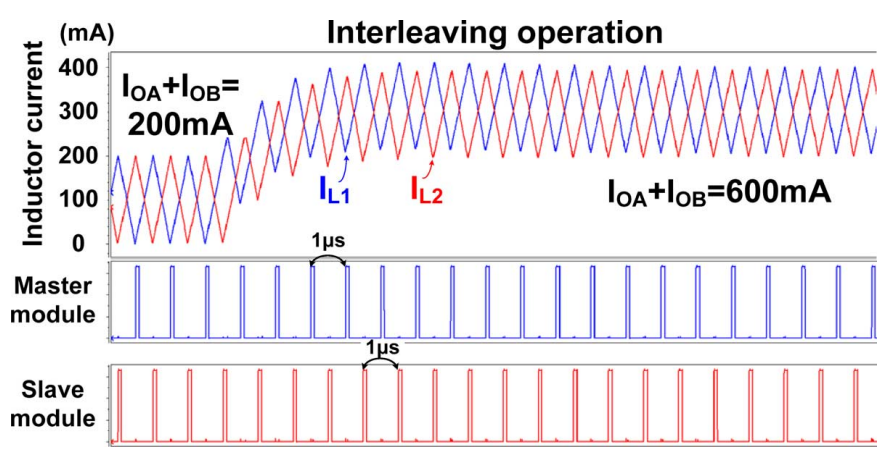

Fig. 12. The interleaving operation with the IECM control in the proposed SIDO modules.

current matching in load transient response and the operation of the phase shift circuit.

\section{EXPERIMENTAL RESULTS}

This proposed SIDO converter with the IECM control was fabricated by $65 \mathrm{~nm}$ CMOS technology. The measured waveforms of the single-phase operation are shown in Figs. 13-15. Fig. 13 demonstrates the single-phase operation in steady-state with $V_{O A}$ of $1.8 \mathrm{~V}$ and $V_{O B}$ of $1.2 \mathrm{~V}$. The energy delivery paths

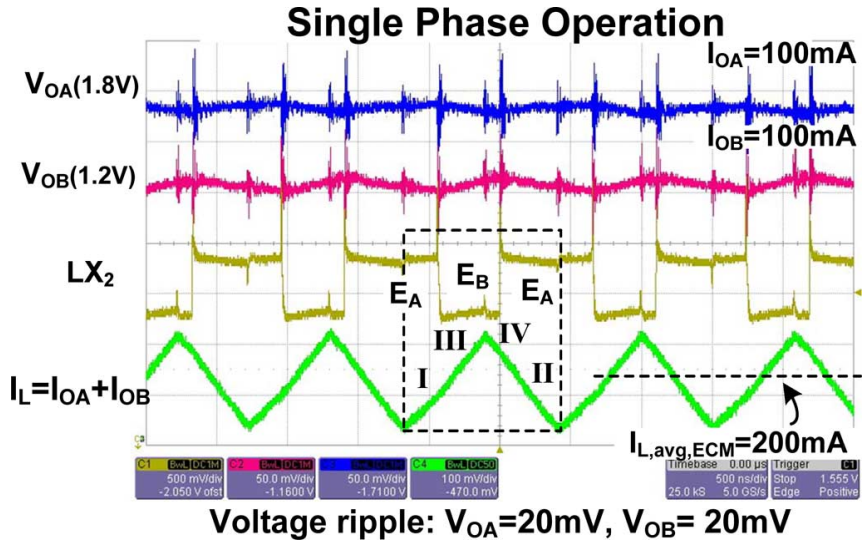

Fig. 13. Measured single-phase operation of ECM control with $\mathrm{I}_{O A}=$ $100 \mathrm{~mA}$ and $\mathrm{I}_{O B}=100 \mathrm{~mA}$.

in the ECM control are verified through the occurrence of path-I, path-III, path-IV, and path-II in sequence. With a load current of $100 \mathrm{~mA}$ at each output, the average inductor current is continuously maintained at $200 \mathrm{~mA}$, which is equal to the summation of the two output loads. The output voltage ripple is about $20 \mathrm{mV}$ at each output due to the discontinuous inductor current. When each output has $200 \mathrm{~mA}$ load current, the measured single-phase operation with the ECM control is also achieved through the ordered energy delivery paths shown in Fig. 14. Similarly, the average inductor current is equal to the summation of the two output load currents. The output voltage ripple is about $30 \mathrm{mV}$ at each output. Fig. 15 shows the measured load transient response in single-phase operation. The largest output drop voltage is about $80 \mathrm{mV}$, whereas the largest overshoot voltage is about $60 \mathrm{mV}$. The voltage recovery time is derived within $30 \mu \mathrm{s}$. In addition, good voltage regulation and fast transient response are also achieved because of the high dc loop gain and bandwidth. The average inductor current is always equal to the summation of two output loads by the ECM control. Thus, the minimum inductor current level is achieved through the superposition technique to enhance the power conversion efficiency and reduce the output voltage ripple. 


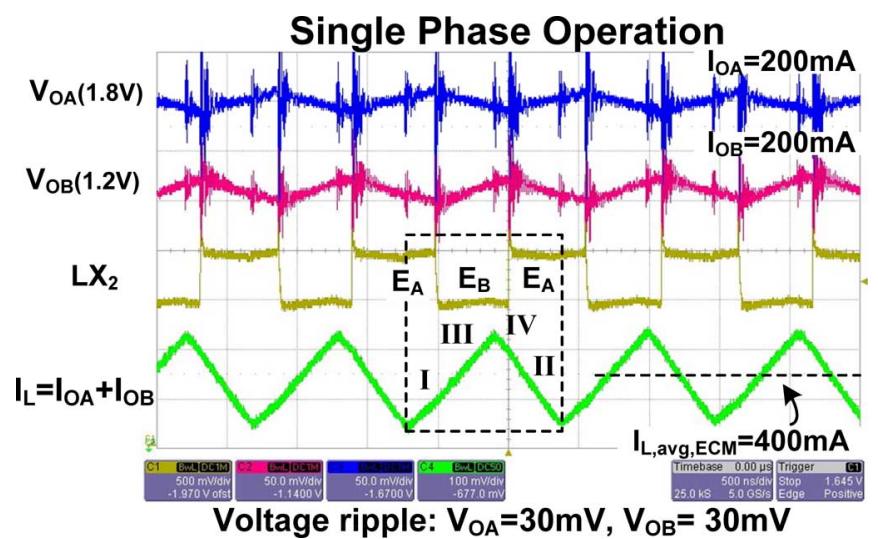

Fig. 14. Measured single phase-operation of ECM control with $\mathrm{I}_{O A}=$ $200 \mathrm{~mA}$ and $\mathrm{I}_{O B}=200 \mathrm{~mA}$.

\section{Single Phase Operation}

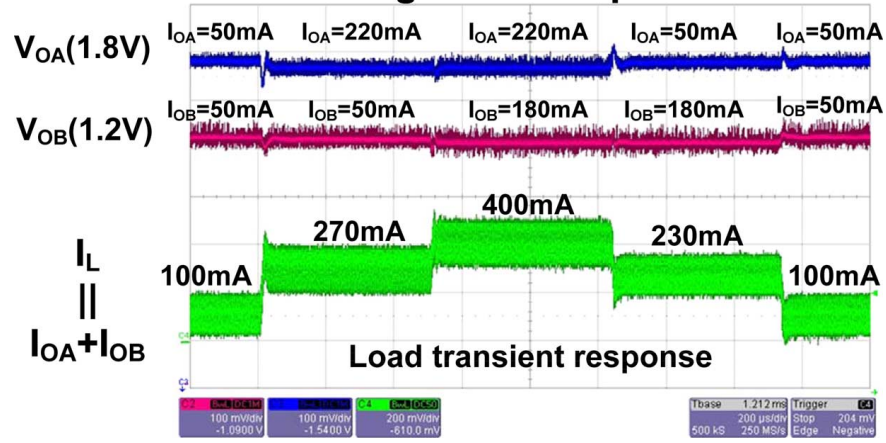

Fig. 15. Measured single-phase operation in case of load transient response.

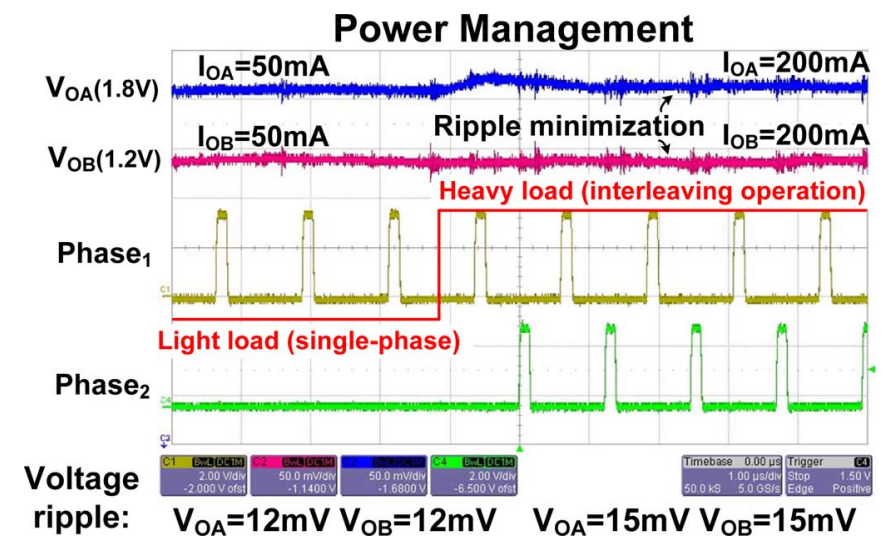

Fig. 16. Measured interleaving operation of the power management integration.

Fig. 16 shows the measured waveform of the power management function. When the UWB system enters the data transmission period, an increase in the power request activated the IECM control to provide a large driving capability simultaneously from the two interleaving SIDO modules. Moreover, owing to the current interleaving mechanism, the output voltage ripple is greatly reduced, and the voltage spike resulting from the ESR is nearly eliminated. The output voltage ripple is reduced to $15 \mathrm{mV}$ at each output with $200 \mathrm{~mA}$ output load current.

The measured power conversion efficiency of the single-phase operation is shown in Fig. 17. The conduc-

\section{Power conversion efficiency}

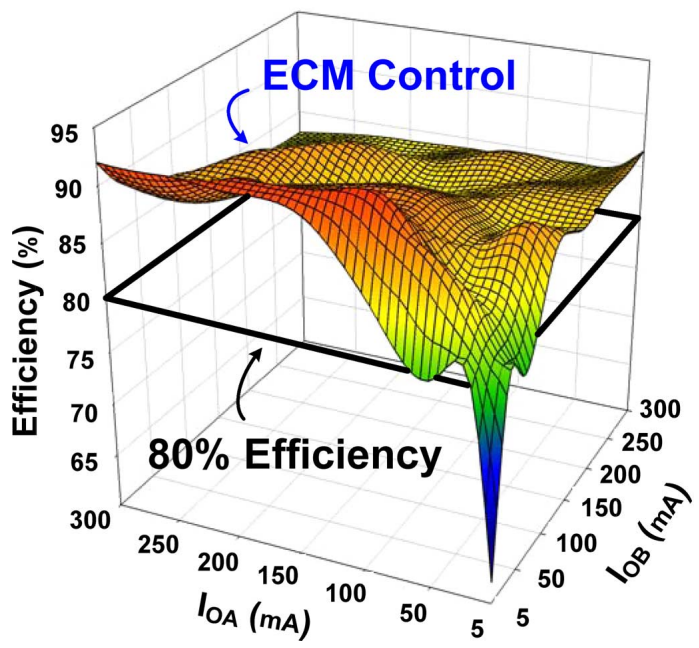

Fig. 17. Measured power conversion efficiency in the single-phase operation with $V_{\mathrm{IN}}=3.3 \mathrm{~V}, V_{O A}=1.8 \mathrm{~V}$, and $V_{O B}=1.2 \mathrm{~V}$.

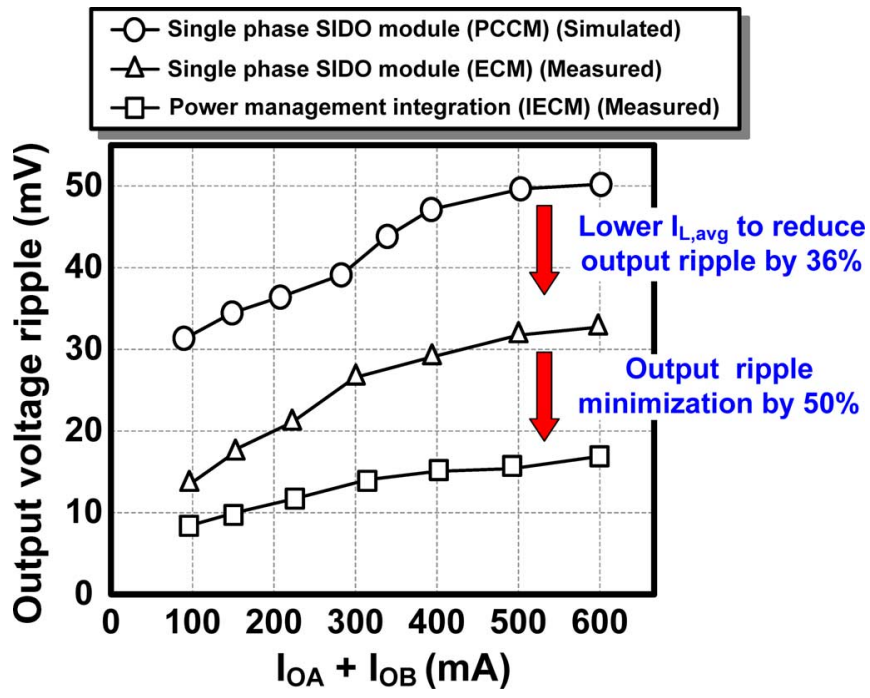

Fig. 18. The comparison of the output voltage ripple by using different control methods with $V_{\mathrm{IN}}=3.3 \mathrm{~V}, V_{O A}=1.8 \mathrm{~V}$, and $V_{O B}=1.2 \mathrm{~V}$.

tion loss of the power switches can be greatly reduced owing to the proposed ECM control. Peak efficiency increases to $91 \%$, where the $80 \%$ efficiency is achieved by today's commercial products with four power switches. The comparison of the output voltage ripple with different loads is shown in Fig. 18. Output voltage ripple in the ECM control decreases by as much as $36 \%$ compared with that in the PCCM control. Specifically, a 50\% reduction in the output ripple is presented at heavy loads because of the current interleaving with the IECM control. The design specifications of the proposed SIDO converter are listed in Table II. Moreover, the comparisons of the prior SIDO methodologies are shown in Table III.

Fig. 19 shows a chip micrograph and the illustration of a 3-D package. The occupied silicon area of the single SIDO module is $1.44 \mathrm{~mm}^{2}$. The inductor is placed on the top of the power management ICs to shorten the distance of the bounding wire. The performance is enhanced through the alleviation of the bond wire effect since the length of the bonding wire is short- 
TABLE II

DESIGN SPECIFICATIONS OF THE PROPOSED SIDO CONVERTER

\begin{tabular}{|c|c|c|c|c|}
\hline Process & \multicolumn{4}{|c|}{$65 \mathrm{~nm}$ CMOS process } \\
\hline Input voltage & \multicolumn{4}{|c|}{$2.7 \mathrm{~V}-3.6 \mathrm{~V}$} \\
\hline Switching frequency & \multicolumn{4}{|c|}{$1 \mathrm{MHz}$ (nominal) } \\
\hline $\begin{array}{c}\text { Chip size } \\
\text { (SIDO module) }\end{array}$ & \multicolumn{4}{|c|}{$1600 \mu \mathrm{m}$ x $900 \mu \mathrm{m}$} \\
\hline Modules & \multicolumn{2}{|c|}{ Master module } & \multicolumn{2}{|c|}{ Slave module } \\
\hline Inductor / DCR & \multicolumn{2}{|c|}{$4.7 \mu \mathrm{H} / 250 \mathrm{~m} \Omega$ (nominal) } & \multicolumn{2}{|c|}{$4.7 \mu \mathrm{H} / 250 \mathrm{~m} \Omega$ (nominal) } \\
\hline Outputs & $\mathrm{V}_{\mathrm{OA}}=1.8 \mathrm{~V}$ & $\mathrm{~V}_{\mathrm{OB}}=1.2 \mathrm{~V}$ & $\mathrm{~V}_{\mathrm{OC}}=1.5 \mathrm{~V}$ & $\mathrm{~V}_{\mathrm{OD}}=1 \mathrm{~V}$ \\
\hline Output capacitor / ESR & $4.7 \mu \mathrm{F} / 30 \mathrm{~m} \Omega$ & $4.7 \mu \mathrm{F} / 30 \mathrm{~m} \Omega$ & $4.7 \mu \mathrm{F} / 30 \mathrm{~m} \Omega$ & $4.7 \mu \mathrm{F} / 30 \mathrm{~m} \Omega$ \\
\hline \multirow{2}{*}{ Cross Regulation } & $\begin{array}{l}0.1 \mathrm{~A} \rightarrow 0.3 \mathrm{~A} \\
0.3 \mathrm{~A} \rightarrow 0.1 \mathrm{~A}\end{array}$ & $\begin{array}{c}10 \mathrm{mV} \\
8 \mathrm{mV}\end{array}$ & $\begin{array}{l}0.1 \mathrm{~A} \rightarrow 0.3 \mathrm{~A} \\
0.3 \mathrm{~A} \rightarrow 0.1 \mathrm{~A}\end{array}$ & $\begin{array}{c}12 \mathrm{mV} \\
6 \mathrm{mV}\end{array}$ \\
\hline & $\begin{array}{l}12 \mathrm{mV} \\
15 \mathrm{mV}\end{array}$ & $\begin{array}{l}0.1 \mathrm{~A} \rightarrow 0.3 \mathrm{~A} \\
0.3 \mathrm{~A} \rightarrow 0.1 \mathrm{~A}\end{array}$ & $\begin{array}{l}11 \mathrm{mV} \\
12 \mathrm{mV}\end{array}$ & $\begin{array}{l}0.1 \mathrm{~A} \rightarrow 0.3 \mathrm{~A} \\
0.3 \mathrm{~A} \rightarrow 0.1 \mathrm{~A}\end{array}$ \\
\hline $\begin{array}{l}\text { Output ripple } \\
\text { (Single phase) }\end{array}$ & \multicolumn{4}{|c|}{$<32 \mathrm{mV}$} \\
\hline $\begin{array}{c}\text { Output ripple } \\
\text { (Interleaving operation) }\end{array}$ & \multicolumn{4}{|c|}{$<16 \mathrm{mV}$} \\
\hline
\end{tabular}

TABLE III

COMPARISON OF THE PREVIOUS SIDO (SIMO) METHOdOLOGIES

\begin{tabular}{|c|c|c|c|c|c|}
\hline & $\begin{array}{c}\text { This work } \\
\text { (ECM control) }\end{array}$ & $\begin{array}{c}\text { CICC } 2009 \\
{[3]}\end{array}$ & $\begin{array}{c}\text { JSSC } 2003 \\
{[4]}\end{array}$ & $\begin{array}{c}\text { JSSC } 2009 \\
{[8]}\end{array}$ & $\begin{array}{c}\text { CICC 2009 } \\
{[10]}\end{array}$ \\
\hline Technology & $65 \mathrm{~nm} \mathrm{CMOS}$ & $\begin{array}{c}0.35 \mu \mathrm{m} \\
\mathrm{CMOS}\end{array}$ & $\begin{array}{l}0.5 \mu \mathrm{m} \\
\mathrm{CMOS}\end{array}$ & $\begin{array}{c}0.25 \mu \mathrm{m} \\
\mathrm{CMOS}\end{array}$ & $\begin{array}{c}0.25 \mu \mathrm{m} \\
\mathrm{CMOS}\end{array}$ \\
\hline Supply voltage & $2.7-3.6 \mathrm{~V}$ & $1.8-2.4 \mathrm{~V}$ & $1.25-2.25 \mathrm{~V}$ & $1.8-2.2 \mathrm{~V}$ & $2.7-5 \mathrm{~V}$ \\
\hline Switching frequency & $1 \mathrm{MHz}$ & $1.25 \mathrm{MHz}$ & $1 \mathrm{MHz}$ & $660 \mathrm{kHz}$ & $1.3 \mathrm{MHz}$ \\
\hline Outputs & 2 Buck & 2 Boost & 2 Boost & $\begin{array}{l}2 \text { Buck, } \\
2 \text { Boost }\end{array}$ & 2 Buck \\
\hline Inductor & $4.7 \mu \mathrm{H}$ & $1 \mu \mathrm{H}$ & $1 \mu \mathrm{H}$ & $10 \mu \mathrm{H}$ & $4.7 \mu \mathrm{H}$ \\
\hline Output capacitor & $4.7 \mu \mathrm{F}$ & $4.7 \mu \mathrm{F}$ & $33 \mu \mathrm{F}$ & $33 \mu \mathrm{F}$ & $47 \mu \mathrm{F}$ \\
\hline Output voltage ripple & $<32 \mathrm{mV}$ & $<160 \mathrm{mV}$ & $<25 \mathrm{mV}$ & $<22 \mathrm{mV}$ & $<30 \mathrm{mV}$ \\
\hline Load current (max.) & $0.3 \mathrm{~A} / 0.3 \mathrm{~A}$ & $0.4 \mathrm{~A} / 0.2 \mathrm{~A}$ & $0.12 \mathrm{~A} / 0.1 \mathrm{~A}$ & N/A & $0.25 \mathrm{~A} / 0.26 \mathrm{~A}$ \\
\hline Transient voltage drop & $\begin{array}{c}80 \mathrm{mV} \\
\left(\Delta \mathrm{I}_{\text {load }}=0.17 \mathrm{~A}\right)\end{array}$ & $\begin{array}{c}500 \mathrm{mV} \\
\left(\Delta \mathrm{I}_{\text {load }}=0.2 \mathrm{~A}\right)\end{array}$ & N/A & N/A & $\begin{array}{c}36 \mathrm{mV} \\
\left(\Delta \mathrm{I}_{\text {load }}=0.27 \mathrm{~A}\right)\end{array}$ \\
\hline Transient settling time & $\begin{array}{c}25 \mu \mathrm{s} / \\
\left(\Delta \mathrm{I}_{\text {load }}=0.17 \mathrm{~A}\right)\end{array}$ & $\begin{array}{c}100 \mu \mathrm{s} \\
\left(\Delta \mathrm{I}_{\text {load }}=0.2 \mathrm{~A}\right)\end{array}$ & N/A & N/A & $\begin{array}{c}10 \mu \mathrm{s} \\
\left(\Delta \mathrm{I}_{\text {load }}=0.27 \mathrm{~A}\right)\end{array}$ \\
\hline Load-regulation & $<10 \%$ & $<20 \%$ & N/A & $<2 \%$ & $<5 \%$ \\
\hline Cross-regulation & $<8 \%$ & $<10 \%$ & N/A & $<0.35 \%$ & $<5 \%$ \\
\hline Peak efficiency & $91 \%$ & $87.8 \%$ & $89.4 \%$ & $93 \%$ & $87 \%$ \\
\hline Chip area & $1.44 \mathrm{~mm}^{2}$ & $2.21 \mathrm{~mm}^{2}$ & $4.25 \mathrm{~mm}^{2}$ & $3.78 \mathrm{~mm}^{2}$ & $5.29 \mathrm{~mm}^{2}$ \\
\hline
\end{tabular}

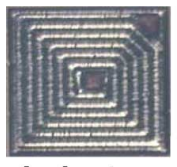

Inductor
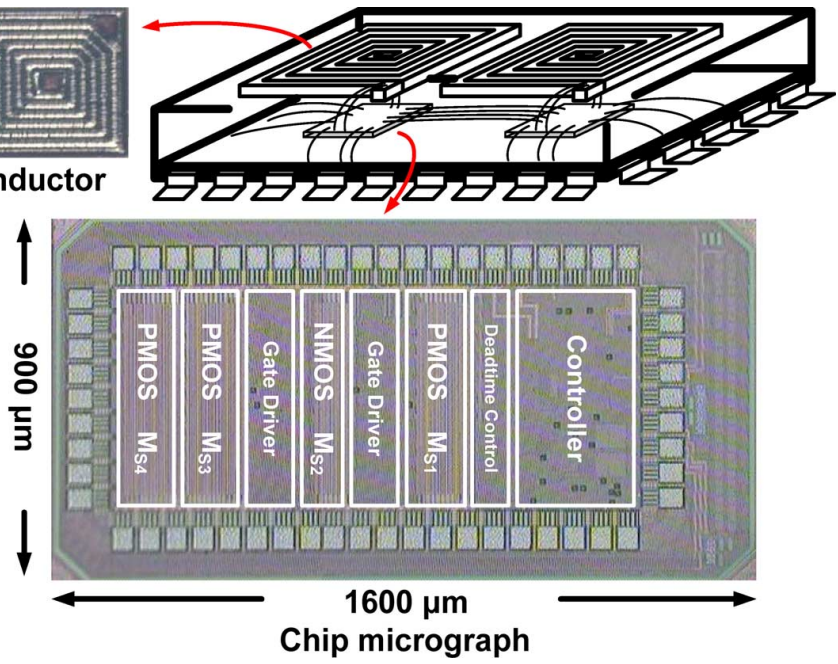

Fig. 19. The chip micrograph and the illustration of inductor integration.

ened as well as the equivalent bond wire inductance. It minimizes the switching noise and voltage spike resulting from the

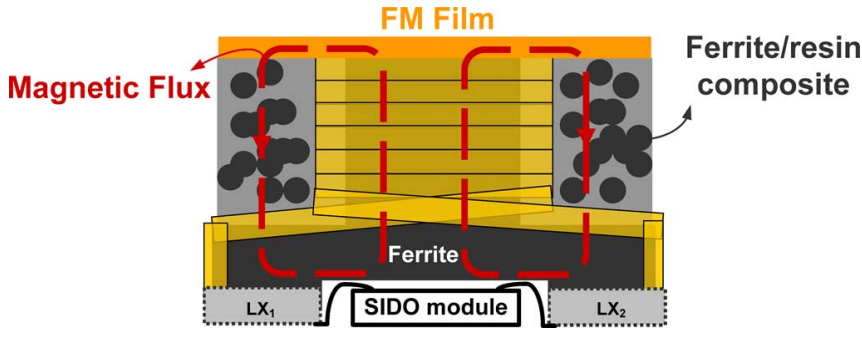

Fig. 20. The details of the inductor integration.

power stage. The details of the inductor integration are given in Fig. 20. Chip function is affected by the magnetic flux generated from the inductor. However, a magnetic flux produced by conductive coil cannot be blocked by insulating it from its surrounding materials [18]. Nevertheless, the magnetic field can be a short-circuited in place of the high permeability magnetic material, which provides an easy path with low reluctance for the return magnetic flux to form a closed magnetic circuit. Therefore, the magnetic flux would propagate along the high permeability material, which is composed of the ferrite and fer- 
rite-magnet (FM ) film and ferrite/resin composite, to largely decrease the interference to the chip function. In addition, relative to the ferrite and FM material, the permeability of air is low. Thus, the leakage flux and the magnetic field interference from the inductor can be minimized. Consequently, the integration of the inductor with the proposed SIDO converter achieves the area-efficient power management module and enhances the competitiveness to the UWB system or other system-on-a-chip applications.

\section{CONCLUSION}

The proposed SIDO converter with IECM control was fabricated by $65 \mathrm{~nm}$ CMOS technology. The ECM control is achieved through the ordered energy delivery paths to power the UWB system. Besides, the superposition technique helps derive a minimum average inductor current, which is equal to the summation of two output loads. In addition, ICEM control is activated to realize the interleaving operation with current interleaving. Thus, a larger supply power can be provided during the data transmission period, and the output voltage ripple can be reduced owing to the current interleaving mechanism. Experimental results demonstrate the single-phase and interleaving operations of the proposed SIDO converter. A peak efficiency of $91 \%$ is achieved and the output voltage ripple appears notably minimized by over $50 \%$ through current interleaving at heavy load. The test chip occupies $1.44 \mathrm{~mm}^{2}$ and integrates with a 3-D architecture for inductor integration.

\section{REFERENCES}

[1] H.-P. Le, C.-S. Chae, K.-C. Lee, S.-W. Wang, G.-H. Cho, and G.-H. Cho, "A single-inductor switching DC-DC converter with five outputs and ordered power-distributive control," IEEE J. Solid-State Circuits, vol. 42, no. 12, pp. 2076-2714, Dec. 2007.

[2] K.-S. Seol, Y.-J. Woo, G.-H. Cho, G.-H. Cho, J.-W. Lee, and S.-I. Kim, "Multiple-output step-up/down switching DC-DC converter with vestigial current control," in IEEE ISSCC Dig. Tech. Papers, 2009, pp. 442-443.

[3] X. Jing, P. K. T. Mok, and M. C. Lee, "A wide-load-range single-inductor dual-output boost regulator with minimized cross-regulation by constant-charge-auto-hopping (CCAH) control," in Proc. IEEE Custom Integrated Circuits Conf. (CICC), 2009, pp. 299-302.

[4] D. Ma, W.-H. Ki, and C.-Y. Tsui, "A pseudo-CCM/DCM SIMO switching converter with freewheel switching," IEEE J. Solid-State Circuits, vol. 38, no. 6, pp. 1007-1014, Jun. 2003.

[5] M.-H. Huang and K.-H. Chen, "Single-inductor dual buck-boost output (SIDBBO) converter adaptive current control mode (ACCM) and adaptive body switch (ABS) for compact size and long battery life in portable devices," in IEEE Symp. VLSI Circuits Dig., 2009, pp. 164-165.

[6] M. Belloni, E. Bonizzoni, E. Kiseliovas, P. Malcovati, F. Maloberti, T. Peltola, and T. Teppo, "A 4-output single-inductor DC-DC buck converter with self-boosted switch drivers and 1.2 A total output current," in IEEE ISSCC Dig. Tech. Papers, 2008, pp. 444-445.

[7] C.-S. Chae, H.-P. Le, K.-C. Lee, G.-H. Cho, and G.-H. Cho, "A singleinductor step-up DC-DC switching converter with bipolar outputs for active matrix OLED mobile display panels," IEEE J. Solid-State Circuits, vol. 44, no. 2, pp. 509-524, Feb. 2009.

[8] M.-H. Huang and K.-H. Chen, "Single-inductor multi-output (SIMO) DC-DC converters with high light-load efficiency and minimized cross-regulation for portable devices," IEEE J. Solid-State Circuits, vol. 44, no. 4, pp. 1099-1111, Apr. 2009.

[9] E. Bonizzoni, F. Borghetti, P. Malcovati, F. Maloberti, and B. Niessen, "A $200 \mathrm{~mA}$ 93\% peak efficiency single-inductor dual-output DC-DC buck converter," in IEEE ISSCC Dig. Tech. Papers, 2007, pp. 526-527.
[10] W. Xu, Y. Li, X. Gong, Z. Hong, and D. Killat, "A single-inductor dual-output switching converter with low ripples and improved cross regulation," in Proc. IEEE Custom Integrated Circuits Conf. (CICC), 2009, pp. 303-306.

[11] K.-H. Chen, C.-J. Chang, and T.-H. Liu, "Bidirectional current-mode capacitor multipliers for on-chip compensation," IEEE Trans. Power Electron., vol. 23, no. 1, pp. 180-188, Jan. 2008.

[12] C. F. Lee and P. K. T. Mok, "A monolithic current-mode CMOS DC-DC converter with on-chip current-sensing technique," IEEE J. Solid-State Circuits, vol. 39, no. 1, pp. 3-14, Jan. 2004.

[13] H.-W. Huang, K.-H. Chen, and S.-Y. Kuo, "Dithering skip modulation, width and dead time controllers in highly efficient DC-DC converters for system-on-chip applications," IEEE J. Solid-State Circuits, vol. 42, no. 11, pp. 2451-2465, Nov. 2007.

[14] Y. Qiu, X. Chen, and H. Liu, "Digital average current-mode control using current estimation and capacitor charge balance principle for DC-DC converters operating in DCM," IEEE Trans. Power Electron., vol. 25 , pp. 1537-1545, Jun. 2010.

[15] K. Viswanathan, R. Oruganti, and D. Srinivasan, "A novel tri-State boost converter with fast dynamics," IEEE Trans. Power Electron., vol. 17, pp. 677-683, Sep. 2002.

[16] Y.-H. Lee, Y.-Y. Yang, K.-H. Chen, Y.-H. Lin, S.-J. Wang, K.-L. Zheng, P.-F. Chen, C.-Y. Hsieh, Y.-Z. Ke, Y.-K. Chen, and C.-C. Huang, "A DVS embedded power management for high efficiency integrated SoC in UWB system," IEEE J. Solid-State Circuits, vol. 45, no. 11, pp. 2227-2238, Nov. 2010.

[17] R. W. Erickson and D. Maksimović, Fundamentals of Power Electronics. Norwell, MA: Kluwer, 2001.

[18] C. H. Ahn and M. G. Allen, "Micromachined planar inductors on silicon wafers for MEMS applications," IEEE Trans. Ind. Electron., vol. 45, no. 6, pp. 866-876, Dec. 1998.

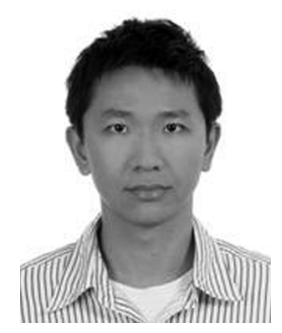

Yu-Huei Lee (S'09) was born in Taipei, Taiwan. $\mathrm{He}$ received the B.S. and M.S. degrees from the Department of Electrical and Control Engineering, National Chiao Tung University, Hsinchu, Taiwan, in 2007 and 2009, respectively. He is currently pursuing the Ph.D. degree in the Institute of Electrical Control Engineering, National Chiao Tung University, Hsinchu, Taiwan.

$\mathrm{He}$ is a Faculty Member at the Mixed Signal and Power Management IC Laboratory, Institute of Electrical Control Engineering, National Chiao Tung University, Hsinchu, Taiwan. His current research interests include the power management integrated circuit design, light-emitting diode driver IC design, and analog integrated circuits.

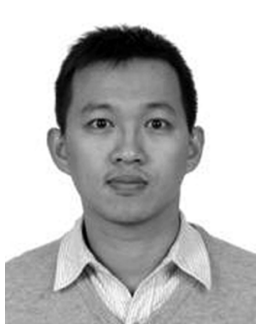

Yao-Yi Yang (S'09) was born in Changhua, Taiwan. He received the B.S. and M.S. degrees from Chung Yuan Christian University and National Taipei University of Technology in 2004 and 2007, respectively. He is currently pursuing the Ph.D. degree in the Institute of Electrical Control Engineering, National Chiao Tung University, Hsinchu, Taiwan.

$\mathrm{He}$ is a member of the Mixed Signal and Power Management IC Laboratory at National Chiao Tung University. His research interests include the power the analog integrated circuits. management IC design, LED driver IC design and,

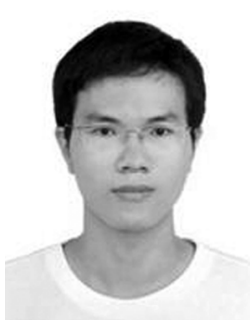

Shih-Jung Wang (S'09) was born in Taipei, Taiwan. He received the B.S. and M.S. degrees from the Department of Electrical and Control Engineering, National Chiao Tung University, Hsinchu, Taiwan, in 2007 and 2009, respectively.

$\mathrm{He}$ is a member of the Mixed Signal and Power Management IC Laboratory at National Chiao Tung University. His research interests include the design of power management circuit, LED driver ICs, and the analog integrated circuit designs. 


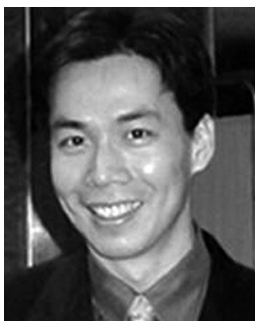

Ke-Horng Chen (M'04-SM'09) received the B.S., M.S., and Ph.D. degrees in electrical engineering from National Taiwan University, Taipei, Taiwan, in 1994, 1996, and 2003, respectively.

From 1996 to 1998, he was a part-time IC Designer at Philips, Taipei. From 1998 to 2000, he was an Application Engineer at Avanti, Ltd., Taiwan. From 2000 to 2003, he was a Project Manager at ACARD, Ltd., where he was engaged in designing power management ICs. He is currently an Associate Professor in the Department of Electrical Engineering, National Chiao Tung University, Hsinchu, Taiwan, where he organized a Mixed-Signal and Power Management IC Laboratory. He is the author or coauthor of more than 80 papers published in journals and conferences, and also holds several patents. His current research interests include power management ICs, mixed-signal circuit designs, display algorithm and driver designs of liquid crystal display (LCD) TV, red, green, and blue (RGB) color sequential backlight designs for optically compensated bend (OCB) panels, and low-voltage circuit designs.

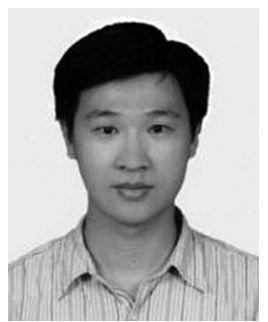

Ying-Hsi Lin received the B.S. degree from National Chiao-Tung University, Hsinchu, Taiwan, in 1993, and the M.S. degree in electrical engineering from National Taiwan University in 1995.

$\mathrm{He}$ joined the Computer and Communication Research Lab at ITRI as a researcher in 1995, and became project leader of CMOS RF and high speed mixed-signal circuits design in 1998. Since joining ITRI CCL, he has been working on CMOS radio frequency integrated circuits and mixed-signal circuits IC design for computer and communication application. In October 1999, He joined Realtek Semiconductor Corp., as an RF manager, where he was responsible for several R\&D CMOS RF projects including Bluetooth, WLAN 802.11abg, 802.11n, WLAN CE and UWB, and also involving CMOS RF IC mass production planning. In the circuits design, his activities ranged are RF synthesizer, LNA, Mixer, modulator, PA, filter, PGA, mixed-signal circuits, ESD circuits, RF device modeling, RF system calibration and communication system design. In 2010, he became the Vice President of Realtek and led the Research and Design Center. He holds more than 30 patents in the area of mixed-signal and RF IC design.

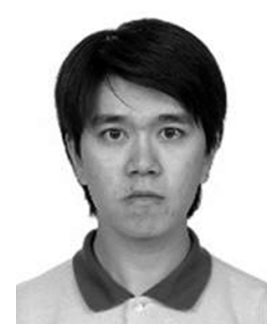

Yi-Kuang Chen received the B.S. and M.S. degrees from National Cheng Kung University, Tainan, Taiwan, in 2003 and 2005, respectively.

He joined Realtek Semiconductor Corporation in September 2005 as a circuit designer. He is currently involved in analog and mixed-signal circuits design. His research interests include line drivers and switching regulators for SoC.

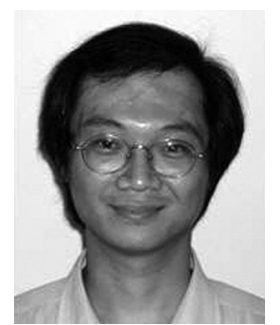

Chen-Chih Huang received the B.S. degree from National Chiao-Tung University, Hsinchu, Taiwan, in 1990, and the M.S. degree in electrical engineering from National Taiwan University, Taipei, Taiwan, in 1992.

He joined Mosel Vitelic Inc., Hsinchu, as an engineer in 1994. In 1995, He joined Realtek Semiconductor Corp., Hsinchu, as an analog circuit design engineer. During 1995-2010, he was responsible for several projects including fast Ethernet/Gigabit Ethernet network interface controller/ PHYceiver/ switch controller, Clock generator, USB, ADSL router, Gateway controller, etc. He is currently the Senior Manager of the Analog_CN design team of the R\&D center. 